\title{
Exploring the Design of Social VR Experiences with Older Adults
}

\author{
Steven Baker \\ School of Computing and \\ Information Systems, The \\ University of Melbourne \\ Melbourne, Australia \\ steven.baker@unimelb.edu.au
}

\author{
Jenny Waycott \\ School of Computing and \\ Information Systems, The \\ University of Melbourne \\ Melbourne, Australia \\ jwaycott@unimelb.edu.au
}

\author{
Romina Carrasco \\ School of Computing and \\ Information Systems, The \\ University of Melbourne \\ Melbourne, Australia \\ rcarrasco@student.unimelb.edu.au
}

\author{
Thuong Hoang \\ School of Information \\ Technology, Deakin University \\ Geelong, Australia \\ thuong.hoang@deakin.edu.au
}

\author{
Frank Vetere \\ School of Computing and \\ Information Systems, The \\ University of Melbourne \\ Melbourne, Australia \\ f.vetere@unimelb.edu.au
}

\begin{abstract}
There is growing interest in technologies that allow older adults to socialise across geographic boundaries. An emerging technology in this space is social virtual reality (VR). In this paper we report on a series of participatory design workshops that involved extended in-depth collaboration with 22 older adults (aged 70-81), that aimed to understand their views on the types of social VR experiences that they saw as being of value to older adults. This process culminated in a reminiscence-based social VR concept. Our study identifies: participants' ideas about the types of social VR experiences they found appealing; the potential for social VR as a powerful reminiscence tool; how social VR might be used as a tool to challenge ageing stereotypes and promote healthy ageing. Reflecting on the design process, we discuss how the diverse participant groups and the complexities involved in mediating between designers, the technical team, and participants could inform future design work.
\end{abstract}

\section{Author Keywords}

Older Adults, Virtual Reality, Participatory Design, Reminiscence

\section{CCS Concepts}

-Human-centered computing $\rightarrow$ Human computer interaction (HCI);

\section{INTRODUCTION}

It is commonly understood that demographic shifts occurring within many nations will result in an increase in the percentage classroom use is granted without fee provided that copies are not made or distributed for profit or commercial advantage and that copies bear this notice and the full citation on the first page. Copyrights for components of this work owned by others than the author(s) must be honored. Abstracting with credit is permitted. To copy otherwise, or republish, to post on servers or to redistribute to lists, requires prior specific permission and/or a fee. Request permissions from permissions@acm.org.

DIS'19, Designing Interactive Systems Conference 2019, June 23-28, 2019, San Diego, CA, USA

(C) 2019 Copyright held by the owner/author(s). Publication rights licensed to ACM. ISBN 978-1-4503-5850-7/19/06...15.00

DOI: $10.1145 / 3322276.3322361$ of the older adult population [61]. This realisation has led to a sharp increase in political and research interest into how best to fund and implement services and supports that allow older adults to age well $[6,2,50]$. While there are many aspects of healthy ageing $[35,63]$, a well known issue is the detrimental effect of social isolation on well-being [52]. In responding to the challenges associated with social isolation, HCI researchers have increasingly been concerned with the design of technologies such as mobile/tablet applications $[25,59,2]$ and social networking services $[2,56,16]$ that will allow older adults to remain socially connected, even if other age-related factors limit their ability to participate fully in their local communities.

Virtual reality (VR) is an emerging social technology which has demonstrated promising potential as a medium for social connections [37]. An obvious point of difference between VR and existing social technology is the fully immersive quality of VR: wearing head-mounted displays, users can enter and interact within a shared three dimensional virtual world. However, little is known about the potential for this technology to support social interactions among older people. Given the importance of social participation for older adults, our study sought to understand older adults' views on social virtual reality through a series of participatory design workshops.

This work builds on an earlier six-month study with a group of older adults (aged between 70 and 81) that introduced them to a range of VR technologies, supported them to design their first embodied avatars - digital self-representations that will be controlled via natural body movements and gestures [41], and provided them with the opportunity to interact in two social VR environments $[3,11]$. This paper reports on findings and experiences from subsequent participatory design workshops with the same cohort. In these workshops we: 1) conducted focus group sessions with 22 older adults to understand the types of social VR applications they believed would support meaningful social participation, 2) asked the same participant groups to comment on and critique 
three social VR scenarios that were developed in response to the focus group data, and 3) collaborated with our participants to refine a social VR concept that focused on shared reminiscence.

In this paper we tell the story of this collaboration and discuss how the study contributes to our understanding of how social VR might play a positive role in older adults lives. Our paper makes a number of core contributions: 1) our findings detail the social VR experiences our participants felt would be most engaging to older adults and we discuss how these insights might inform future design work, 2) we discuss the critical role that reminiscence played for our participants and the unique advantages social VR applications have as a reminiscence tool, 3) we describe how social VR applications and the avatars used in these virtual worlds were viewed by our participants as tools that challenge ageing stereotypes and support healthy ageing, and 4) we reflect on the participatory process itself and detail a number of aspects of our study that were designed to address issues of power with respect to decision making, and how our decision making might change for future studies based on this reflection.

\section{PRIOR WORK}

\section{Older Adults and VR}

There is growing research interest in the design and application of VR to specific challenges facing older people, particularly in the areas of rehabilitation and dementia care. The study of VR as a tool to assist with rehabilitation for older adults has received significant attention within the health-care and gerontological literature $[22,36,46]$. Key areas of evaluation relate to the use of VR and exergames as a tool to improve range of movement [36], and the application of VR to specific conditions such as Parkinson's disease [22].

There has also been considerable interest in the application of VR for those living with dementia [18, 32, 38, 48]. Hodge et al. [18], report on a series of design workshops focused on designing "novel virtual reality environments for people with dementia and their carers" [p.10]. Though not designing social VR experiences, they note that participants in the study saw VR as a tool that could support connections between carers and older adults [18]. Though this finding hints at the potential for VR to be used as a social medium, with the exception of our earlier work [3, 11], we are unaware of any other studies examining the potential of VR as a social tool with older users.

\section{Older Adults and Social Virtual Worlds}

While the design of social VR applications with older users is a nascent area of research, a body of literature has examined the use of social virtual worlds (such as Second Life). These applications differ from social VR in that they are not fully immersive environments accessed via head-mounted displays, but rather provide the opportunity to interact in virtual worlds accessed via a computer or tablet device.

Reed and Fitzpatrick [45] conducted a number of workshops with 22 older adults examining their use of the social virtual world Second Life. Though primarily focused on self-representation and the design of avatars, their findings suggest that some older adults were motivated to use Second Life as a means of communicating with distant relatives, a goal that was thwarted by the limited "possibilities afforded by the Second Life software" (p.168). Siriaraya and Ang [49] conducted a study examining a desktop PC based 3D virtual world that allowed pairs of older participants to control avatars and take part in a collaborative shopping task. Results showed that the participants "found 3D avatars to be of limited use to their social interaction" (p.114). They suggest that avatars with more realistic features and being able to control the avatar using natural gestures may "improve avatar-mediated communication for older users" (p.114). While limited, this prior work suggests that while older adults were interested in the types of communication that can be achieved in social virtual worlds, they seek more realistic and immersive experiences than those afforded by desktop-based social virtual worlds.

\section{Older Adults and Online Communication}

While there is limited research into the use of social virtual worlds with older adults, a significant body of research has considered older adults use of online social networks. This work has identified a number of issues, such as concerns about privacy and anonymity $[10,19,34]$, and a desire for simplified interfaces $[13,30]$ that have been shown to be particularly important to older adults using online social technologies. In considering future design goals for social networks, Hope and colleagues have demonstrated that older adults value "deeper, well thought out, carefully crafted social communications" and modalities that "enable depth of thought, reflection, and personalization" [19, p.3911]. This finding is supported by similar work that has suggested those designing for late life development should design online communication systems that "support complex and multifaceted identities of older adults" [9, p.9]. This social network focused research demonstrates the unique communication needs of older adults. To meet these needs, researchers and designers must seek to integrate older adults into the design process in order to ensure that their desire for personalised and complex forms of communication are 'baked in' to any social communication tool.

\begin{abstract}
AIMS
Recent research by Moustafa and Steed [37] examining small group interactions in VR with younger users (average age 30.4) found that the immersive nature of social VR "evoke(d) a more humanistic interaction between users" and that the sense of presence evoked by the technology allowed "users to interact naturally with one another in VR" (p.8). These findings suggest that social VR may address many of the limitations that prevent older users from embracing other social technologies by offering a space where older adults can interact naturally in personalised virtual worlds that support the types of deeper, reflective forms of communication that has been demonstrated as being valued by this cohort $[10,19,13,30,9]$. However, research is yet to fully explore whether and how VR can be designed to support meaningful social interaction among older people. Therefore, in this study we aim to explore, together with older adults, how VR might provide new opportunities for mediated social interactions that satisfy their unique communication needs.
\end{abstract}

\section{METHODS}

Below, we provide a description of the participants before detailing the structure of the participatory design workshops that comprised this study, and the methods used to analyse the workshop data. 


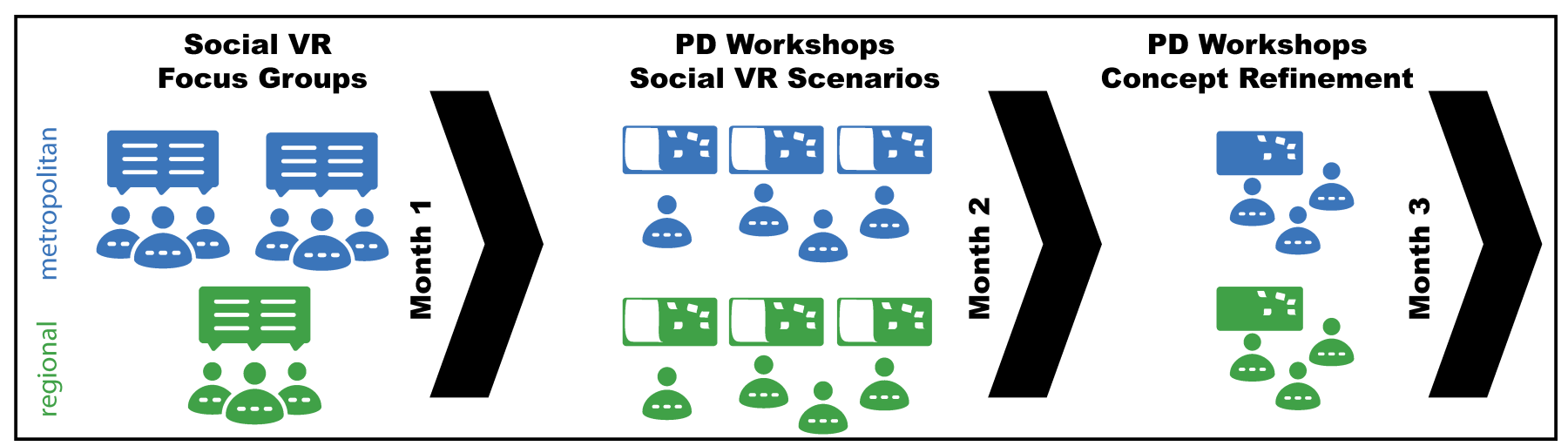

Figure 1. Figure showing the three distinct phases of the study

\section{Participants}

Of the 25 participants initially recruited, 22 agreed to take part in our study (8 regional, 14 metropolitan). The regional group comprised four male and four female participants, while the metropolitan group consisted of six female and eight male participants. The participants were aged between 70 and 81. All were retired or semi-retired having worked in a wide range of professions including as homemakers, teachers and academics, salespeople, and healthcare workers. Most were living independently in the community or in retirement communities except for 'Cleo' who lived in a residential aged care facility.

Though most participants were able bodied, some used walking frames to assist with mobility issues, several participants had hearing aids, and one participant was living with advanced Parkinson's Disease. The only inclusion criteria for recruiting participants was that they were over the age of 70 and were interested in new technologies. While many participants had some experience with computing technology, none reported having used VR or an avatar prior to the initial study. Pseudonyms will be used when describing the participants' contributions during the study.

While there was a pool of 22 participants involved with the study, attendance at individual workshop sessions varied considerably. Two factors in particular impacted on participant numbers. First, the workshops took place over the winter months, a time when several of our participants went on vacation to warmer climates. A second factor that particularly impacted on the second workshop was that it was held during a school holiday period where a number of participants were unavailable as they had responsibilities caring for grandchildren. Participant numbers for each phase of the study were; Social VR Focus Groups (Regional $=7$, Metropolitan =13), Social VR Scenario Workshops (Regional = 3 , Metropolitan $=10$ ), Concept Refinement Workshops (Regional $=6$, Metropolitan $=12$ ).

\section{Workshop Setting}

The study took place in workshop rooms at regional and metropolitan university campuses. These were chosen as they provided adequate space, were available at a reasonable expense, and had access to catering facilities. The latter proved to be a very important aspect of the study as the shared lunch held at the conclusion of each workshop provided a 'neutral space' where researchers and participants could discuss the workshops informally.

\section{Workshop Program}

A design team consisting of the first and third authors were responsible for planning and conducting the workshops that comprised each phase of the study in both the regional and metropolitan areas. In the intervening period between study phases, the design team held weekly meetings with a technical team who would be responsible for developing a prototype application based on the outcomes of the study. These meetings involved discussing key outcomes from each phase and planning for upcoming workshops. Participants were encouraged to attend these meetings either in person or via video conference, however, Samuel was the only participant to accept this invitation.

Two members of the technical team also attended the metropolitan 'Social VR Scenario' and 'Concept Refinement' workshops. This prompted impromptu 'VR masterclasses' where some of the more technically curious metropolitan participants posed questions about VR technology such as: how are the systems able to detect and interpret body movements?; how powerful do the computers need to be to run a VR program? This impromptu aspect of the study was valued by both the metropolitan participants and the technical team.

The workshop program was split across three distinct phases. The nature of each phase is described below and outlined in figure 1.

\section{Social VR Focus Groups:}

The first phase of the study consisted of focus group sessions which were followed by a shared lunch. Having recently completed the preceding study, the participants were primed to give their views on VR systems and to discuss the potential of VR to support social participation. We therefore decided that facilitated focus group discussions would be an ideal way of capturing this feedback. A small number of focus group questions were prepared that were focused on gaining a better understanding of what types of social experiences the participants thought would be enjoyable. As the participants were by this stage very familiar with VR technology and with each other, little prompting was required and the focus groups quickly centred on social VR ideas that were of interest to the participants.

\section{Social VR Scenarios:}

The second phase of the study consisted of two participatory design workshops (one regional, one metropolitan) held approximately one month after the social VR focus groups. In these workshops which lasted approximately 90 minutes (followed by 
a shared lunch), participants critiqued three social VR scenarios that had been developed by the design team. The scenarios were created after analysing the results of the focus groups, and after meetings with the technical team. Each scenario described a fictitious social VR application that acted as a provocation for the participants to consider the design characteristics they believed would be most important in social VR. At the end of the workshop, participants were asked to rank each scenario from most favourite to least favourite and add comments using Post-it notes about the pros and cons of each scenario. Members of the research team circulated around the participants as they evaluated each scenario and took notes about their discussions with the participants.

\section{Concept Refinement:}

The third and final phase of the study consisted of two further participatory design workshops (one with the regional group and one with the metropolitan group) held approximately one month after the presentation of the social VR scenarios. This workshop also lasted 90 minutes and was followed by a shared lunch. After a short feedback session where the results of the previous workshops were discussed, participants were asked to reappraise the most popular scenario from the social VR scenario workshops and refine the concept so as to make sure that it responded to their needs. Feedback collected during the session included Post-it notes with comments from the participants, and researcher notes from their discussions with participants.

\section{Data Collection and Analysis}

A range of qualitative data was collected during each phase of the study including: research notes taken by members of the design team, audio recordings, photographs, and Post-It notes that were contributed by the participants during the final two phases of the study. These data were transcribed verbatim where necessary and imported into NVivo software for further analysis. Data were then subjected to thematic analysis using Braun and Clarke's six-stage analytic approach [8]. A copy of the document detailing each stage of the Braun and Clarke process (completed by the first author) was sent to the entire research team so that all could interrogate and comment on the thematic process as a coding consistency check [27].

As workshops developed in response on the results of the previous focus groups or workshops, results and key themes will be presented chronologically below.

\section{SOCIAL VR FOCUS GROUPS}

Three focus groups were held during this phase, one based in the regional setting $(\mathrm{n}=7)$ and two in the metropolitan setting $(\mathrm{n}=7$, $\mathrm{n}=6$ ). The settings were $150 \mathrm{~km}$ apart. Splitting the metropolitan group in two allowed for more intimate group discussion. The focus groups each lasted approximately 30-40 minutes followed by a shared lunch, and all followed the same basic structure consisting of a small number of semi-structured prompts that acted as 'jumping off points' for discussion amongst the groups. Figure 2 shows participants continuing their discussion over lunch at the conclusion of one of the focus groups. After reviewing these open ended discussions, we found that three broad categories of social VR applications were seen as appealing to participants: 1) indoor/outdoor exercise or sports based applications; 2) social club or group based applications; and 3) travel based applications.

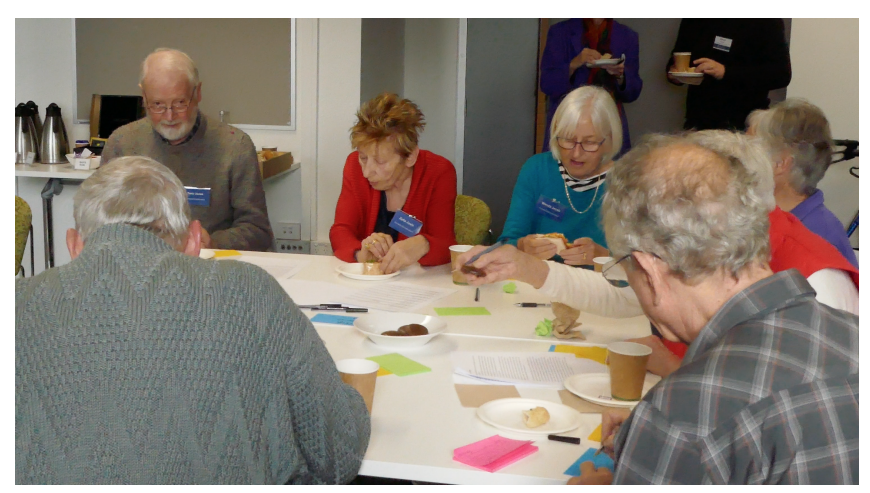

Figure 2. Focus group discussion over lunch

Each of these categories and the individual application ideas are detailed in Table 1. Below, we discuss some of the central themes that permeated the focus group discussions.

\section{Facilitation in Social VR}

Regardless of the categories of social VR applications being discussed, a theme running through much of the conversation was that social experiences are improved when there is an expert facilitator to mediate discussion and provide guidance. When discussing group exercise, Carl suggested that any social VR based exercise application should incorporate an expert trainer who "could facilitate doing exercise, but doing it properly." The idea of being instructed to 'do an exercise properly' resonated with many group members across the focus groups. Several participants discussed interactions with health-care professionals who had given them exercises to perform without adequate guidance on how to do them correctly. Group discussion of social clubs or groups centred around the view that these activities were improved by having an 'expert' who facilitates the discussion. Participants frequently spoke about the need to have someone who "knows more about (the topic) than the rest of the group", or someone who might "open the conversation up or expand people's thoughts." This category of ideas was more explicitly linked to social interaction than the sports/exercise category. While discussing the idea of a social VR travel application, participants focused on the idea of an expert guide who might "show me the hidden parts of Paris" or where "an expert in Van Gogh is showing you around" a virtual gallery.

The theme of facilitation running through the focus group discussion provides insights into some unique ways in which those designing social VR experiences for older adults might improve socialisation through facilitation. There is an established body of research investigating the use of exergames with older adults as a means of encouraging healthy ageing [2]. Participants' valued the idea of social VR exergames that could be facilitated by an expert trainer who can ensure that the exercises are being performed correctly. This type of exercise experience would also address their frustration at being given exercise regimes by health-care professionals without being provided with a means to evaluate their performance. Research into older adults design preferences in relation to social media have established that older adults prefer personalised modalities that enable depth of thought and reflection $[9,19]$. Results form our focus groups suggest that facilitation is valued by older adults as it enables this type of deeper social experience. 
Table 1. Workshop participants' ideas for social VR applications

\begin{tabular}{|c|c|}
\hline Social VR Application Categories & Application Ideas \\
\hline 1) Outdoor Group Exercise/Sports & Bush-Walking; Cycling; Tennis; Golf; Hockey; Rock Climbing \\
\hline Indoor Group Exercise & Gym classes; Dancing Lessons \\
\hline 2) Social Clubs or Social Groups & Dinner party; Card games; Community Choir; Cafe Socrates; Painting \\
\hline 3) Travel & $\begin{array}{l}\text { Guided tour of Paris; Walking tour of Mt Everest; Guided tour of Van } \\
\text { Gough Museum; Tour the Vatican }\end{array}$ \\
\hline
\end{tabular}

\section{Social VR and the Ageing Body}

When discussing social VR exercise activities, many participants linked the desire for a group exercise application to ideas of maintaining independence, what we might term a 'use it or lose it' concept of healthy ageing. Carl typified this view saying he would be interested in any application "that helps us remain independent and responsible for (our) own decisions health wise". The idea of a social VR exercise application was also appealing to some group members, especially women, because of the anonymity that would be afforded by being able to embody an avatar in a social VR exercise application. Sally, who said she spoke to a lot of women about exercise, commented that "a lot of women of roughly my age don't want to go into the public where they can be seen, either because they think their body is flabby and ugly, or because they look old." When discussing social group based VR applications, the potential anonymity or safety of interacting as an avatar in a VR application was also a key topic of discussion. Evan felt these types of group activities, when translated into VR, could open up new opportunities for involvement from people who might otherwise struggle with group interaction. During a discussion of an idea for a social club he commented "taking that into virtual reality, if you are afraid of being seen by someone else, if they are an avatar or something like that, then maybe they might be able to express themselves a bit more than they would if you were looking at their face."

The popularity of the idea of a social VR travel application seemed to be linked to broader factors related to ageing such as adapting to the limitations of an ageing body. Gordon provided a good example of how a social VR travel application might allow you to transcend physical limitations:

They have been advertising a trip... to the base camp of Everest. Now I'm not going to do that, I'm not going to kid myself that I'm fit enough... but it would be interesting (if) instead of seeing someone's slides of a trip, to actually see as much as you can in (VR), I'd love to do that!

Samuel spoke about how life circumstances can impact on long held travel plans. He noted that as his wife had become increasingly disabled in her seventies and that "places that we thought we might travel, we can't".

These results indicate the complex ways in which issues relating to the ageing body, whether rooted in lived experience or negative social stereotypes, influenced our participants views on the benefits of social VR. Previous research has established that older adults can become socially isolated because of stereotypical attitudes that equate the ageing body with decrepitude [23]. Insights from our participants suggest that social VR exercise applications were viewed by some as a means to protect themselves from negative social stereotypes while maintaining the social and physical benefits of group exercise. Results also highlight the role that social VR can play in protecting anonymity. Researchers examining the online communication preferences of older adults have noted that anonymity is particularly valued by older users [10, 19, 34], our participants' comments suggest that social VR is seen as a communication medium that can address these concerns while still offering rich immersive experiences. Finally, our results demonstrate that our participants viewed social VR as a tool that could enable older adults to take part in virtual travel experiences that would otherwise be inaccessible to them. This opens new possibilities for designers to create vivid travel experiences, and interrogate new interaction techniques, that allow older users with different levels of physical ability to not just view a new destination, but share this experience with others.

\section{Reminiscence}

A final theme that was evident in much of the focus group discussion was the idea that social VR might provide opportunities to reminisce in new and interesting ways. It was clear that some participants saw exercise applications as offering the chance to re-experience the 'thrill' of an exercise activity without the risk of physical injury. Samuel recalled riding a bike down a mountainside and the feeling "about halfway down... that I am a teenager again" before stating that he "wouldn't dare to now, partly because the consequences (if he fell) are so much greater." Kim commented that although she had been a talented hockey player, she had to leave that part of her life behind, stating I don't want to play hockey anymore, too many bruises. "Len summarised the groups feelings about re-experiencing former sporting glories by commenting they were "probably an indication that we're at an age where a lot of sporting activities are much more difficult now, and we reminisce". There was a lot of discussion amongst participants about reminiscence and their desire to pass on knowledge. Klaus regretted that much of his technical expertise was no longer valued because of technological change. Reflecting on his short wave radio skills he commented that "people are just not interested in what they can't see an application for." Len echoed these sentiments. "In the last 20 or 30 years, a whole lot of things that we were very conversant with are no longer appropriate, no longer utilised." When asked if this knowledge of previous technology might give older adults a unique perspective on the design of new technology, Len responded "Yes, we have a naivete in that we're coming from a different point in discovery." Participants also discussed the challenges of capturing and passing on knowledge. Carl spoke about the frustration of attending a funeral only to learn about a fascinating aspect of the 
deceased person's life that you never knew. Len regretted that his grandchildren did not seem interested in talking "about things that used to be". Tina responded "I have noticed in my family that you skip a generation... the next generation's extremely interested and they want to know everything... but two generations before are dead, and you've got to try and remember things that you've been told and you (often were not) told much."

Reminiscence therapy is a common technique employed by carers, especially in aged-care settings. Recent research had begun to consider how VR might be used as a reminiscence tool [18], however, research to date has focused predominantly on viewing "generic media" and asking older adults to reflect on the memories such footage elicits. Our findings suggest a much more vibrant and active type of reminiscence therapy is possible, one that leverages the immersive properties of social VR to provide older adults with opportunities to collaboratively (re)experience thrilling activities within a safe environment. The strong theme of reminiscence that pervaded conversations during the focus groups highlights how important reminiscence is to older adults and the potential that technology can play in promoting reminiscence [24]. One interesting feature of the reminiscence discussion that took place during the focus groups is the idea that reminiscing about technological change is a means by which older users might bring unique insights into the design space by assessing design, as Len put it, 'from a different point in discovery'. The next workshops would be our chance to tap into this unique perspective as we sought to develop a social VR application concept with our participants.

\section{SOCIAL VR SCENARIOS}

After reflecting on the discussions that took place in the focus groups, the design team created three social VR scenarios to be introduced in the next participatory design workshops that were presented as provocations. These workshops were held approximately one month after the focus groups. Two workshops were held, one regional $(n=3)$ and one metropolitan $(n=10)$. A2-sized posters describing each scenario were posted around the workshop space accompanied by large sheets of blank paper. Using markers and coloured Post-It notes, the participants posted comments and recommendations on how the scenarios could be improved, before ranking them from most to least favourite. An image of a participant viewing one of the scenarios during this workshop can be seen in figure 3 .

\section{Scenario Descriptions}

The three scenarios drew on insights from the focus groups in varying ways. A table summarising the outline of each scenario is presented in Table 2.

Card Shark - The first scenario presented a social VR concept based on the idea of a social card game that had been a popular topic during the focus groups. A core concept underpinning this scenario was collaboration and the ability to incorporate a facilitator to encourage socialisation as the game progressed.

Mt Arapiles - This scenario drew on the the popularity of outdoor activities and travel in the focus groups. The design team hoped the scenario would encourage cooperation and social interaction while also providing a 'thrill-seeking' element. The scenario drew directly on themes from the focus groups including having a role

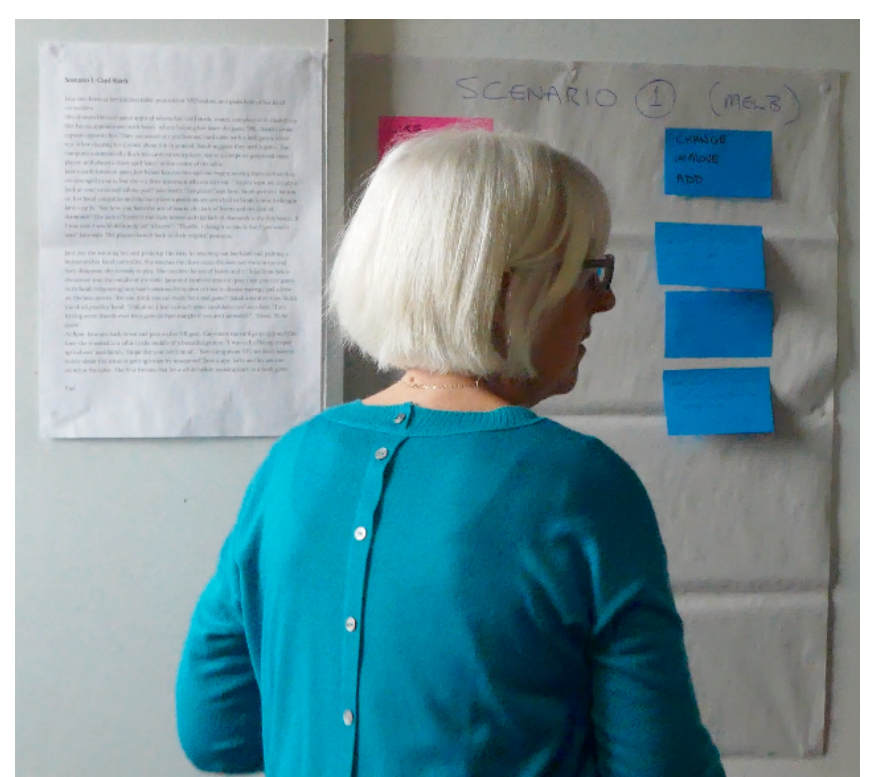

Figure 3. Participant viewing a scenario during the first participatory design workshops

for a climbing instructor (facilitator), and adopting a positive view of healthy ageing that challenged negative stereotypes.

Highway of Life - This scenario was inspired by the many references to reminiscence and capturing life experiences that permeated discussion in the focus groups. The scenario proposed a social application that would allow participants to step back in time by walking down a metaphorical highway of life and 'stopping off' at historical points along the way to discuss their life experiences.

\section{Participant Reactions to the Scenarios}

\section{Card Shark:}

Reactions to this scenario were the most varied, with sharp divisions existing between those who already enjoyed card games and those who did not. Gordon's comment illustrates the attitude of the non-card playing group "I am not a card player, so this doesn't have any built-in appeal for me... I guess if you were an ex card fiend you might think differently." Of those who liked the scenario, there was support for the card game to be Bridge as it was considered more "popular with older people." Participants suggested the game would be improved by incorporating differing skill levels and training options. The scenario offered the card players a choice of virtual environments in which they could play and the participants felt this was an advantage of a VR game that would prompt players to discuss their surroundings. Another common comment was that participants wanted the ability to customise their avatar in this type of social VR application. Evan commented that 'Card Shark' was the "most likely scenario where I would not like to look like myself, maybe (I could) even look like a shark!" Participants felt the ability to customise their avatar would allow them to deceive the other players to gain a strategic advantage. The most positive comments relating to the scenario centred on its ability to incorporate social interaction and learning into a single application. 


\begin{tabular}{ll}
\hline Scenario Name & Scenario Summary \\
\hline Scenario One 'Card Shark' & $\begin{array}{l}\text { Social VR card game that allows "Jane" to meet with her friends "Sarah", "Luke" and "Jay" } \\
\text { in various social environments to play the card game 500. }\end{array}$
\end{tabular}

Scenario Two 'Mt Arapiles' "Greg" chooses his sporty avatar before meeting "Sally" at the base of Mt Arapiles. They must work together to navigate their way up the face of the mountain, sharing tips and tricks to find the best 'holds' but also race each other to be the first to the top. At the top of the mountain they are rewarded with a stunning view and can discuss their climb and the beautiful scenery

Scenario Three "Highway of "David", "Gen", and "Lisa" are transported to the middle of a highway that stretches out Life' to the horizon. Signposts beside the road indicate the years as they stretch back in time. The group teleports to 1969 and interact with historical artefacts that prompt them to recall their personal stories of the moon landing.

\begin{abstract}
Mt Arapiles:
Despite its more adventurous premise, 'Mt Arapiles' was less divisive than 'Card Shark' with only one participant (Wes) labelling it as definitely not his "cup of tea." Although comments were generally more positive toward this scenario, there were a number of specific concerns raised relating to the likelihood that the application would induce vertigo, an issue that is especially relevant in the context of VR [46, 22, 29, 40]. The only other negative comments related to the competitive element that was present in the scenario where users would 'race each other to the top'. Several participants questioned this premise and instead argued that it would be "better to emphasise the achievement of actually 'doing it'." Many seemed attracted to the 'thrill' of the scenario. Cleo compared it to an earlier life experience of hang gliding and overcoming her fear "I was sure I'd have a heart attack, but gradually I began to soar... adrenalin rush. I DID IT." When asked what she liked about the 'Mt Arapiles' scenario, Violet said "It's different! Defeats 'old age' stereotypes."
\end{abstract}

\section{Highway of Life:}

Scenario three 'Highway of Life' solicited the most positive feedback. Gordon's comment was illustrative of the positive reaction to the scenario: "I like the general applicability of this scenario. Everyone can go back in time in their memory and be challenged to recollect their personal experiences of the particular era chosen." One of the most intriguing aspects of the comments were those related to the idea of using the 'Highway of Life' to imagine what might have been if alternative decisions were made. These feelings were especially prevalent in the metropolitan group. Amy, Evan, and Herbert all made comments of this type, such as Evan's "What if (a) decision made in the past had been different, would my life have been better or worse?" Herbert wondered if his avatar might be able to consider the "possibilities of alternate pathways and choices." Amy felt the scenario would allow users to reflect on experiences and explore "what made us who we are." The positive reaction to the scenario was perhaps most clearly articulated by Klaus in the regional group who simply wrote "This is right on the ball." The only participant to express any reservation about the scenario was Carl in the metropolitan group who commented that while others may be interested in "looking back", he personally preferred to "lookforward".

\section{Scenario Preferences}

The 'Highway of Life' was the most preferred application scenario in both the regional and metropolitan groups (regional average 1.3, metropolitan 1.4). Carl was the only participant to rank the 'Highway of Life' as the least favourite scenario, whereas both 'Card Shark' and 'Mt Arapiles' had multiple participants rank them as their least favoured option. The regional group gave both 'Mt Arapiles' and 'Card Shark' the same average ranking (2.3). Average rankings from the metropolitan group were similar ('Mt Arapiles' 2.3 and 'Card Shark' 2.1).

A clear outcome from the second phase findings was that the Highway of Life scenario was the application idea that most resonated with the group. When reflecting on this and the generally positive reaction to the Highway of Life scenario in the month after the workshops concluded, the design team decided to conduct one more set of participatory design workshops before commencing the development of a Highway of Life inspired prototype.

\section{CONCEPT REFINEMENT}

The concept refinement workshops provided an opportunity for the participants to engage more deeply with the Highway of Life concept and participate directly in adapting the concept so that it would reflect their social VR preferences. As with the preceding phase, two workshop sessions were held, one regional $(n=6)$ and one metropolitan $(n=12)$. The sessions began with a recap of the results from the second workshop to provide participants with the rationale for choosing to proceed with development of the Highway of Life concept.

At the end of this introductory discussion, we followed a similar process to the last participatory design workshops and attached large sections of blank paper to the walls around the workshop space. After being given markers and Post-It notes, participants were asked to respond to a number of questions relating to the Highway of Life concept. To narrow down the focus for development, the first question asked 'What five-year period of your life would you most like to revisit in the Highway of Life?' The second and third questions were much more general: 'How will the Highway of Life support social interaction?', and 'What are the most important design considerations for the Highway of Life?'. Members of the research team were also on hand to record comments made by participants as they discussed the 
scenario and commented on the questions. In the sections below, we describe the participants' comments and reactions to the concept refinement questions.

What five year period of your life would you most like to revisit in the Highway of Life?

Responses to the first question were split into two clusters. In the first, participants accepted the premise of the question and provided (roughly) five-year periods which they felt were the most important. Most of these centred on the mid-1950s to the late 1960s, a time span that corresponded to most participants' adolescence and early adulthood. Some participants commented that the reason for making this choice related to the political and social changes that occurred during this time. For others, particularly in the regional group, the reasons for choosing their time period were more personal. Mandy's comment is illustrative:

1958-1965 - I was eighteen years old. Eighteen for me was lovely, old enough to be treated as an adult and still be able to do silly kids stuff. Old enough to have a car and get your licence. I had a little blue escort van. I also had my own hairdressing salon. These were exciting years. I met my future husband (in) 1956, married when I was 21 in 1961. We built our first home in 1961 and we had our first son in 1962.

The second cluster of responses pointed out a major flaw with the design team's idea of designing for a five-year period. As the participants ranged in age between 70 and 81 , significant time periods, such as school years, were spread out to the extent that no five-year period could capture a common shared experience. To solve this flaw, rather than choosing a five-year period, participants proposed a focus on life experiences. Six participants across the two workshops made this suggestion and pointed to key life experiences including "school years", "beginning work", "marriage/children", and "retirement".

How will the Highway of Life support social interaction? Many participant responses focused on the need to allow people using the application to interact and, as Gordon put it, "Bounce things off one another." Rather than focusing on the events of the time, participants were more interested in "sharing memories (and) getting to know more about the persons accompanying you" in the Highway of Life. In order to facilitate this personal sharing, participants were united in recommending they be able to bring personal artefacts into the environment, rather than being forced to watch grand historical events. Amy suggested these artefacts could be clustered around common shared activities or scenarios such as, "school", "clothes", "music", and "family dynamics". Others offered ideas ranging from sharing personal "photos, movies... and written records" from the era, or importing local "newspaper clippings". Rather than photographs, Gordon was interested in the ability to share physical objects such as "ink wells", "school prizes" or "school bags" that would prompt users to compare their school experiences.

What are the most important design considerations for the Highway of Life?

Participants used this question to highlight what they saw as a major flaw in the scenario. Whereas the design teams scenario had proposed a video of the moon landings as a feature that would inspire reminiscence, participants advocated instead for a design focus on capturing the contours of individual experience. Herbert neatly summarised this sentiment by saying the application should "discuss and recall (the) minutiae of life, not major events which are already documented." Amy advocated for the ability to upload photos or short narratives to support the users to learn more about other people's personal stories, while Samuel felt it was important that the conversations that took place in the application should be able to be "take(n) away in segments to share with family and friends." Several participants contributed user experience ideas such as using the metaphor of a "time capsule" to revisit events. Klaus felt that the application could include a "virtual newspaper" and that users could "touch the area of interest and get a detail for viewing/participating." Summarising much of the sentiment of the workshops, Harry advocated for a design that allowed users to "talk about common experiences, relive the feeling of that time, share that experience with others, (and) listen to how others reacted to those same experiences."

\section{DISCUSSION}

While our findings helped us to refine the design goals for the prototype social VR application that would be the ultimate focus of our research project, they also highlight broader learning that can be used to inform the design of future social technologies, aimed at older adults. Chief amongst these are: a range of insights into the types and defining features of social VR experiences that our participants felt would respond to older adults' needs; the potential ways social VR might be used as a powerful reminiscence tool; and the potential for social VR to be used as a tool to challenge ageing stereotypes.

\section{Older Adults' Preferred Social VR Experiences}

While our participants ultimately chose to refine a scenario based on reminiscence, findings from the focus groups highlight a range of social VR experiences that our participants felt would be most appealing to older users. These insights contribute to our knowledge of how to best develop social experiences that resonate with the values of older users.

In proposing a range of applications that responded to both indoor and outdoor group exercise, our participants affirm the positive role that VR can play in healthy ageing. In contrast to earlier work that has focused on the use of VR as a rehabilitation tool $[22,36,46]$, our participants spoke of the potential role that VR can play as a preventative health care tool that can help older users maintain their independence. In particular, our findings demonstrate that our participants saw social VR exercise applications as offering an opportunity to learn how to perform exercises correctly with the help of an expert guide. In fact, the appeal of expert guidance in social VR permeated all categories of social VR experiences that were suggested by our participants.

When discussing VR applications centred around social clubs or groups, our participants highlighted the joy they found in sharing experiences with others and the potential they saw in social VR as a tool to foster these experiences. Amy thought the immersive qualities of social VR might allow her to play Bridge with others in a way that would preserve the dynamic of playing with a real partner, an experience she found lacking in existing online platforms. Our participants also highlighted some ways in which VR might afford richer social experiences than real life by allowing those 
who might otherwise be afraid of being seen by others the opportunity, as Evan put it, to "express themselves a bit more than they would if you were looking at their face". Previous research has noted that some older adults self-isolate due to age-related factors [14]. Our participants suggest that social VR may provide a means by which this group could be supported to reengage in social life.

Travel was another category of applications that had strong appeal to our participants. In recent decades, travel has become an increasingly popular activity for older adults around the world [42]. However, research into older adult tourism suggests that those aged 75 and older are far less likely to travel [31]. Our participants highlighted a range of issues such as fear about terrorism, concerns about increased insurance costs, and ill health, that impacted on their ability to travel. Given these issues, participants felt that social VR applications might provide older adults who face limitations that prevent them from realising their travel aspirations with an opportunity to engage in travel experiences that offered similar levels of immersion with like-minded virtual travel companions with whom they could share their experiences.

\section{Social VR and Reminiscence}

The concept of reminiscence permeated the focus groups and participatory design workshops in both regional and metropolitan contexts. Though reminiscence has been observed in people as young as ten [17], there is a large body of research that has examined the particular importance of reminiscence for older adults. Research into older adult well-being has long identified the benefits of reminiscence or conducting a 'life review' especially in combating loneliness, anxiety and depression [51, $15,47]$. Common non-technological techniques for encouraging reminiscence are group storytelling or 'life review' sessions [20, $47,51]$ and art therapy [44]. Reminiscence has also been a focus within HCI research with the use of photographs, music, memory prompts and 'reminiscence kits' being common technological aids to reminiscence $[24,43,33,5,53,54,25]$.

Our findings add to an emerging body of literature [21, 48] examining the potential for virtual worlds and VR to be used as mediums for reminiscence. However, our study is unique in expanding our understanding about the ways that fully immersive social VR provides unique advantages for reminiscence based interventions. Our participants highlight the potential for social VR to support older adults to share their memories and experiences by using virtual artefacts to trigger these memories. The ability to import fully three dimensional artefacts, such as photographs in frames, school bags, or trophies, and 'pass' these objects between avatars in a fully immersive environment is a particularly exciting design opportunity that is likely to support memory recall [21] and meet older adults' preferences for social technologies that support simple interactions and deep reflection $[13,30,19,9]$. Participants also highlighted the unique ways in which fully immersive social environments might be used to 'reauthor' and reinterpret past experiences, an element of reminiscence that is particularly beneficial [17].

Social VR as a Means of Challenging Ageing Stereotypes Prior research into the design and use of full body avatars has demonstrated their impact on addressing ageing stereotypes [11, 12]. Our study both supports and extends these findings. This was particularly evident in Sally's comments about the role avatars might play in supporting older women to participate in group exercise without fear of judgement.

While prior research has studied the impact of avatars on ageing stereotypes, our findings demonstrate that our participants also saw the social VR experiences themselves as being a powerful means of addressing stereotypes. Our participants proposed social VR applications that allowed them to (re)experience the thrills of adventure sports, support each other to reach shared goals, travel with their partners who had mobility impairments, and participate in a range of activities that helped maintain their independence. Furthermore, research into older adults' communication in social networks has highlighted the importance placed by older adults on social technologies that support privacy, anonymity and multi-faced identities [26, 34, 9]. Our participants felt that social VR offered a number of advantages in relation to privacy, anonymity and identity by allowing users to choose body characteristics or personas that were 'tuned' to particular social experiences such as an exercise class or card game. We expect these insights will support future work examining the positive role that social VR can play in our rapidly ageing societies.

\section{Insights from the Participatory Design Process}

In addition to the learning related to VR for social participation in later life, our engagement with the participants during the focus groups and participatory design workshops has also led to a number of insights about the participatory process that we feel will be of benefit to members of the DIS community. Here we focus on two issues in particular: the mix of metropolitan and regional participants and; the interplay between participants, design, and technical teams.

Involving participants from regional and metropolitan areas: A defining characteristic of our study was its incorporation of participants from both regional and metropolitan areas. We believe the involvement of these two diverse groups added a richness and complexity to our data that would have been reduced had we focused on working with only one group.

We found the regional participants had a particularly strong interest in sharing personal stories and artefacts with the design team. A good illustration of this is Mandy's personal description of why she wanted to explore a particular time period during the concept refinement participatory design workshop. The regional group also went to extraordinary lengths to ensure that they contributed beyond the context of the workshops by sending additional information to the team in follow up emails and by giving the team personal artefacts (such as photo albums and diaries) that they could use as inspiration. Research has demonstrated the importance of social cohesion in rural and regional communities $[1,60,57]$ and we wonder if this played a role in our experiences during the regional workshops.

The metropolitan groups, on the other hand, were distinguished by their intense interest in the mechanics of VR and curiosity about how it might be applied in the future. In several instances, this led to the design team inviting members of the technical team to workshops so they could convene small impromptu 'VR masterclasses' to discuss the technical challenges associated with developing VR applications. 
These digressions and differentiating factors between the regional and metropolitan groups are not always compatible with reporting targeted findings in a research article, but they none-the-less provided important contextual information that we tried to incorporate into the scenarios and final design concept. These experiences have convinced us of the value of incorporating participants from diverse geographic areas and we would encourage others undertaking participatory research to consider the advantages of adopting a similar approach.

\section{The interplay between participants, design, and technical} teams:

Another aspect of the participatory process which is hard to incorporate into a paper of this type, is a full examination of power dynamics present in the interplay between the participants, design team, and in our case, technical team. We note, however, this has been the subject of interest in the participatory design literature [39, 55, 7, 62].

As "design is decision-making" and "decision-making is the exercising of power" [7, p.41], we feel it is important to briefly flag some of the steps we took to address power differentials during the study and our thoughts on a number of decisions that we might reconsider were it possible. We believe this is a particularly important aspect of reflective practice we can undertake given the key members of our design team were relative newcomers to participatory design - what Bannon and colleagues might refer to as "emerging voices" [4].

We took a number of conscious steps during the study that we hoped would offer the participants a greater insight into the decision-making process and address the inherent power differences that could impact on the participatory goals of the project. These included: 1) beginning each workshop with an update to provide the participants with an overview of decisions and deliberation that had occurred within the design and technical meetings that took place in between workshops, 2) Inviting participants to sit in on technical meetings so they would have a chance to participate in these deliberations (an option taken up by one participant), 3) Incorporating a shared lunch into each workshop so that informal conversations about goals and decisions could be raised. While not all of these steps were particularly innovative in-and-of themselves (we note for example that the value of shared meals in participatory work has been the subject of some discussion [28, 58]), we believe that in combination these steps helped to 'lift the curtain' on some hidden aspects of the decision-making process and we aim to keep exploring similar techniques in future studies.

In reflecting on the decisions made during the study we also recognise a number of limitations that we will learn from in conducting future participatory design work. While factors such as time deadlines and budget limitations inevitably impact on the decisions made in developing a study, it would have been helpful to have allowed additional time for the participants to help us to develop the scenarios to be used in the second phase of the study. This process could have led to alternative storyboards for the app ideas that might have added an additional dimension to the second phase of the study. On a related note, while we felt the scenarios were a good way of presenting the 'story' of a basic application concept to stimulate discussion amongst the groups, on reflection, the incorporation of other PD techniques, such as paper prototyping, or drama based techniques [39] might have led to a broader range of design artefacts with which we could have engaged in preparing for the prototype development.

\section{CONCLUSION}

In this study, we partnered with 22 older adults (aged 70 to 81 ) from both regional and metropolitan areas to investigate their views about the potential for social VR applications to serve as tools that can support meaningful social interaction. Using a mixture of focus groups and participatory design workshops over the course of a three month period, our participants identified categories of social VR applications that responded to their social needs, engaged with three social VR scenarios developed from these categories, and refined their most preferred scenario so as to ensure that it responded to their preferences. Our findings illustrated the important role that reminiscence plays in our participants' lives and the advantages they saw in using social VR as a reminiscence tool. Findings also highlight how our participants believed social VR might offer advantages as a medium that can be used to challenge ageing stereotypes and support healthy ageing. In reflecting on the participatory design process, we describe the benefits we saw in engaging with both regional and metropolitan participants, and highlight some of the decision-making processes that informed the study. As a result of conducting this study, we have advanced our understanding about older adults' views on the potential for social VR to be used as a meaningful tool for social participation and have collaborated to define a design concept that uses reminiscence as a medium to support meaningful social interaction.

\section{ACKNOWLEDGEMENT}

This work was supported by the Australian Research Council (DP160101368). We thank the participants for their collaboration, enthusiasm and support for the project. We would also like to thank Zaher Joukhadar for his technical support and leadership during the design workshops. This project was approved by the University of Melbourne's Human Research Ethics Committee.

\section{REFERENCES}

[1] Steven Baker, Jeni Warburton, Suzanne Hodgkin, and Janice Pascal. 2017. The supportive network: Rural disadvantaged older people and ICT. Ageing and Society 37, 6 (2017), 1291-1309.

[2] Steven Baker, Jeni Warburton, Jenny Waycott, Frances Batchelor, Thuong Hoang, Briony Dow, Elizabeth Ozanne, and Frank Vetere. 2018. Combatting social isolation and increasing social participation of older adults through the use of technology: A systematic review of existing evidence. Australasian Journal on Ageing 37, 3 (Sept. 2018), 184-193.

[3] Steven Baker, Jenny Waycott, Frank Vetere, and Thuong Hoang. 2019. The Technology Explorers: Partnering with Older Adults to Engage with Virtual Reality and Virtual Avatars. In Ageing and Digital Technology: Designing and Evaluating Emerging Technologies for Older Adults, Barbara Barbosa Neves and Frank Vetere (Eds.). Springer Singapore, Singapore, 231-246.

[4] Liam Bannon, Jeffrey Bardzell, and Susanne Bødker. 2018. Reimagining Participatory Design-Emerging Voices. ACM 
Transactions on Computer-Human Interaction 25, 1 (Feb. 2018), 1.

[5] Frank R Bentley, Santosh Basapur, and Sujoy Kumar Chowdhury. 2011. Promoting intergenerational communication through location-based asynchronous video communication. Proceedings of the 13th international conference on Ubiquitous computing - UbiComp '11 (2011), 31.

[6] Simon Biggs and Helen Kimberley. 2013. Adult Ageing and Social Policy: New Risks to Identity. Social Policy Report 12, 2 (April 2013), 287-297.

[7] Tone Bratteteig and Ina Wagner. 2012. Disentangling power and decision-making in participatory design. In Proceedings of the 12th Participatory Design Conference: Research Papers - Volume 1. ACM, New York, NY,USA, 41-50.

[8] Virginia Braun and Victoria Clarke. 2006. Using thematic analysis in psychology. Qualitative Research in Psychology. 3, 2 (Jan. 2006), 77-101.

[9] Robin Brewer and Anne Marie Piper. 2016. "Tell it like it really is": A case of online content creation and sharing among older adult bloggers. In Proceedings of the SIGCHI Conference on Human Factors in Computing Systems. ACM, New York, NY, 5529-5542.

[10] Kelly E Caine. 2009. Supporting privacy by preventing misclosure. In CHI'09 Extended Abstracts on Human Factors in Computing Systems. ACM, New York, NY, USA, 3145-3148.

[11] Romina Carrasco, Steven Baker, Jenny Waycott, and Frank Vetere. 2017. Negotiating stereotypes of older adults through avatars. In Proceedings of the 29th Australian Conference on Computer-Human Interaction. ACM, New York, NY, USA, 218-227.

[12] Romina Carrasco, Jenny Waycott, Steven Baker, and Frank Vetere. 2018. Designing the Lost Self: Older Adults' Self-representations in Online Games. In Proceedings of the 2018 Designing Interactive Systems Conference (DIS '18). ACM, New York, NY, USA, 441-452.

[13] José Coelho and Carlos Duarte. 2016. A literature survey on older adults' use of social network services and social applications. Computers in Human Behavior 58 (May 2016), 187-205.

[14] Erin York Cornwell and Linda J Waite. 2009. Social disconnectedness, perceived isolation, and health among older adults. Journal of Health and Social Behavior 50, 1 (March 2009), 31-48.

[15] Jeffrey Cully, Donna LaVoie, and Jeffrey Gfeller. 2001. Reminiscence, personality, and psychological functioning in older adults. Gerontologist 41, 1 (Feb. 2001), 89-95.

[16] Carlos Duarte and José Coelho. 2019. Design of Social Network Services for and with Older Adults. In Ageing and Digital Technology: Designing and Evaluating Emerging Technologies for Older Adults, Barbara Barbosa Neves and Frank Vetere (Eds.). Springer Singapore, Singapore, 307-326.
[17] David Haber. 2006. Life review: implementation, theory, research, and therapy. International Journal of Aging \& Human Development 63, 2 (2006), 153-171.

[18] James Hodge, Madeline Balaam, Sandra Hastings, and Kellie Morrissey. 2018. Exploring the Design of Tailored Virtual Reality Experiences for People with Dementia. In Proceedings of the 2018 CHI Conference on Human Factors in Computing Systems. ACM, New York, NY, USA, 514.

[19] Alexis Hope, Ted Schwaba, and Anne Marie Piper. 2014. Understanding digital and material social communications for older adults. In Proceedings of the SIGCHI Conference on Human Factors in Computing Systems. ACM, New York, NY, USA, 3903-3912.

[20] Nimmi Hutnik, Pam Smith, and Tina Koch. 2012. What does it feel like to be 100 ? Socio-emotional aspects of well-being in the stories of 16 Centenarians living in the United Kingdom. Aging \& Mental Health 16, 7 (June 2012), 811-818.

[21] Francisco Ibarra, Marcos Baez, Francesca Fiore, and Fabio Casati. 2018. Designing for Co-located and Virtual Social Interactions in Residential Care. In Proceedings of the 2018 ACM Conference Companion Publication on Designing Interactive Systems (DIS '18 Companion). ACM, New York, NY, USA, 129-134.

[22] Aram Kim, Nora Darakjian, and James M Finley. 2017. Walking in fully immersive virtual environments: an evaluation of potential adverse effects in older adults and individuals with Parkinson's disease. Journal of NeuroEngineering and Rehabilitation 14, 1 (Feb. 2017), 16.

[23] Debbie Laliberte Rudman. 2015. Embodying positive aging and neoliberal rationality: Talking about the aging body within narratives of retirement. Journal of Aging Studies 34 (Aug. 2015), 10-20.

[24] Amanda Lazar, Hilaire Thompson, and George Demiris. 2014. A systematic review of the use of technology for reminiscence therapy. Health Education \& Behavior 41, 1 Suppl (Oct. 2014), 51S-61S.

[25] Hung-Chi Lee, Ya Fang Cheng, Szu Yang Cho, Hsien-Hui Tang, Jane Hsu, and Chien-Hsiung Chen. 2014. Picgo: Designing Reminiscence and Storytelling for the Elderly with Photo Annotation. In Proceedings of the 2014 Companion Publication on Designing Interactive Systems (DIS Companion '14). ACM, New York, NY, USA, 9-12.

[26] Yu-Hao Lee, Min Xiao, and Robert H Wells. 2018. The Effects of Avatars' Age on Older Adults' Self-Disclosure and Trust. Cyberpsychology, Behavior, and Social Networking 21, 3 (March 2018), 173-178.

[27] June Lennie. 2006. Increasing the rigour and trustworthiness of participatory evaluations: learnings from the field. Evaluation Journal of Australasia 6, 1 (2006), 27-35. 
[28] Ann Light, Sonja Pedell, Toni Robertson, Jenny Waycott, Jeanette Bell, Jeannette Durick, and Tuck Wah Leong. 2016. What's Special About Aging. Interactions 23, 2 (Feb. 2016), 66-69.

[29] James Lin, Henry Duh, Donald Parker, Habib Abi-Rached, and Thomas Furness. 2002. Effects of field of view on presence, enjoyment, memory, and simulator sickness in a virtual environment. In Proceedings IEEE Virtual Reality 2002, Vol. 2002. 164-171.

[30] Siân E Lindley, Richard Harper, and Abigail Sellen. 2009. Desiring to Be in Touch in a Changing Communications Landscape: Attitudes of Older Adults. In Proceedings of the SIGCHI Conference on Human Factors in Computing Systems (CHI '09). ACM, New York, NY, USA, 1693-1702.

[31] Nieves Losada, Elisa Alén, Trinidad Domínguez, and Juan Luis Nicolau. 2016. Travel frequency of seniors tourists. Tourism Manage. 53 (April 2016), 88-95.

[32] Valeria Manera, Emmanuelle Chapoulie, Jérémy Bourgeois, Rachid Guerchouche, Renaud David, Jan Ondrej, George Drettakis, and Philippe Robert. 2016. A Feasibility Study with Image-Based Rendered Virtual Reality in Patients with Mild Cognitive Impairment and Dementia. PLoS One 11, 3 (March 2016), e0151487.

[33] Sheila McCarthy, Heather Sayers, Paul McKevitt, and Mike McTear. 2009. MemoryLane: Reminiscence for older adults. CEUR Workshop Proceedings 499 (2009), 22-27.

[34] Andrew R McNeill, Lynne Coventry, Jake Pywell, and Pam Briggs. 2017. Privacy Considerations when Designing Social Network Systems to Support Successful Ageing. In Proceedings of the 2017 CHI Conference on Human Factors in Computing Systems. ACM, New York, NY, USA, 6425-6437.

[35] Julia Menichetti, Pietro Cipresso, Dario Bussolin, and Guendalina Graffigna. 2015. Engaging older people in healthy and active lifestyles: a systematic review. Ageing Society June (2015), 1-25.

[36] Kimberly J Miller, Brooke S Adair, Alan J Pearce, Catherine M Said, Elizabeth Ozanne, and Meg M Morris. 2014. Effectiveness and feasibility of virtual reality and gaming system use at home by older adults for enabling physical activity to improve health-related domains: a systematic review. Age and Ageing 43, 2 (March 2014), $188-195$.

[37] Fares Moustafa and Anthony Steed. 2018. A Longitudinal Study of Small Group Interaction in Social Virtual Reality. In Proceedings of the 24th ACM Symposium on Virtual Reality Software and Technology (VRST'18). ACM, New York, NY, USA, 22:1-22:10.

[38] Wendy Moyle, Cindy Jones, Toni Dwan, and Tanya Petrovich. 2018. Effectiveness of a Virtual Reality Forest on People With Dementia: A Mixed Methods Pilot Study. Gerontologist 58, 3 (May 2018), 478-487.

[39] Michael Muller and Allison Druin. 2003. Participatory design: the third space in HCI. In Human-computer interaction: Development process, A Sears and J Jacko (Eds.). CRC Press, Poca Raton, FL.

[40] Atsuo Murata. 2004. Effects of duration of immersion in a virtual reality environment on postural stability. International Journal of Human-computer Interaction 2, 906464960 (2004), 961-966.

[41] Kristine L Nowak and Jesse Fox. 2018. Avatars and computer mediated communication: a review of the definitions, uses, and effects of digital representations. Review of Communication Research 6 (2018), 30-53.

[42] Roberto Patuelli and Peter Nijkamp. 2015. Travel Motivations of Seniors: A Review and a Meta-Analytical Assessment. Technical Report. Bologna. 17 pages.

[43] S Tejaswi Peesapati, Victoria Schwanda, Johnathon Schultz, Matt Lepage, So-Yae Jeong, and Dan Cosley. 2010. Pensieve: supporting everyday reminiscence. In Proceedings of the SIGCHI Conference on Human Factors in Computing Systems. ACM, New York, NY, USA, 2027-2036.

[44] R H Ravid-Horesh. 2004. "A temporary guest": the use of art therapy in life review with an elderly woman. The Arts in Psychotherapy 31, 5 (Jan. 2004), 303-319.

[45] Darren J Reed and Geraldine Fitzpatrick. 2008. Acting Your Age in Second Life. In Lecture Notes in Computer Science. 158-169.

[46] Maria T Schultheis and Albert A Rizzo. 2001. The application of virtual reality technology in rehabilitation. Rehabilitation Psychology 46, 3 (2001), 296-311.

[47] Juan Pedro Serrano, Jose Miguel Latorre, Margaret Gatz, and Juan Montanes. 2004. Life review therapy using autobiographical retrieval practice for older adults with depressive symptomatology. Psychology and Aging 19, 2 (June 2004), 270-277.

[48] Panote Siriaraya and Chee Siang Ang. 2014. Recreating living experiences from past memories through virtual worlds for people with dementia. Proceedings of the 32nd annual ACM conference on Human factors in computing systems - CHI '14 (2014), 3977-3986.

[49] Panote Siriaraya and Chee Siang Ang. 2019. The Social Interaction Experiences of Older People in a 3D Virtual Environment. In Perspectives on Human-Computer Interaction Research with Older People, Sergio Sayago (Ed.). Springer International Publishing, Cham, 101-117.

[50] Karin Slegers, Martin PJ Van Boxtel, and Jelle Jolles. 2008. Effects of Computer Training and Internet Usage on the Well-Being and Quality of Life of Older Adults: A Randomized, Controlled Study. The Journals of Gerontology: Psychological Sciences and Social Sciences 63, 3 (2008), 176-184.

[51] Sharifah Munirah Syed Elias, Christine Neville, and Theresa Scott. 2015. The effectiveness of group reminiscence therapy for loneliness, anxiety and depression in older adults in long-term care: a systematic review. Geriatric Nursing 36, 5 (Sept. 2015), 372-380. 
[52] Tina ten Bruggencate, Katrien G Luijkx, and Janienke Sturm. 2017. Social needs of older people: a systematic literature review. Ageing Society May 2017 (2017), 1-26.

[53] Wenn-Chieh Tsai, Daniel Orth, and Elise van den Hoven. 2017. Designing Memory Probes to Inform Dialogue. In Proceedings of the 2017 Conference on Designing Interactive Systems (DIS '17). ACM, New York, NY, USA, 889-901.

[54] Wenn-Chieh Tsai, Po-Hao Wang, Hung-Chi Lee, Rung-Huei Liang, and Jane Hsu. 2014. The Reflexive Printer: Toward Making Sense of Perceived Drawbacks in Technology-mediated Reminiscence. In Proceedings of the 2014 Conference on Designing Interactive Systems (DIS '14). ACM, New York, NY, USA, 995-1004.

[55] John Vines, Rachel Clarke, Peter Wright, John McCarthy, and Patrick Olivier. 2013. Configuring Participation: On How We Involve People in Design. In Proceedings of the SIGCHI Conference on Human Factors in Computing Systems (CHI' 13). ACM, New York, NY, USA, 429-438.

[56] Helena Blažun Vošner, Samo Bobek, Peter Kokol, and Marija Javornik Krečič. 2016. Attitudes of active older Internet users towards online social networking. Computers in Human Behavior 55 (Feb. 2016), 230-241.

[57] Jeni Warburton, Sue Cowan, and Tenzin Bathgate. 2013. Building social capital among rural, older Australians through information and communication technologies: A review article. Australasian Journal on Ageing 32, 1 (March 2013), 8-14.
[58] Jenny Waycott, Sonja Pedell, Frank Vetere, Elizabeth Ozanne, Lars Kulik, Alan Gruner, and John Downs. 2012. Actively engaging older adults in the development and evaluation of tablet technology. In Proceedings of the 24th Australian Computer-Human Interaction Conference. ACM, New York, NY, USA, 643-652.

[59] Jenny Waycott, Frank Vetere, Sonja Pedell, Lars Kulik, Elizabeth Ozanne, Alan Gruner, and John Downs. 2013. Older Adults As Digital Content Producers. In Proceedings of the SIGCHI Conference on Human Factors in Computing Systems (CHI'13). ACM, New York, NY, USA, 39-48.

[60] Rachel Winterton and Jeni Warburton. 2011. Does place matter? Reviewing the experience of disadvantage for older people in rural Australia. Rural Society 20, 2 (April 2011), 187-197.

[61] World Health Organization (WHO). 2015. World Report on Ageing and Health. http:

//www.who.int/ageing/publications/world-report-2015/en/. (2015).

[62] Peter Wright and John McCarthy. 2015. The Politics and Aesthetics of Participatory HCI. Interactions 22, 6 (Oct. 2015), 26-31.

[63] Chien Wen Yuan, Benjamin V Hanrahan, Mary Beth Rosson, and John M Carroll. 2018. Coming of old age: understanding older adults' engagement and needs in coproduction activities for healthy ageing. Behaviour \& Information Technology 37, 3 (March 2018), 232-246. 


\section{University Library}

\section{- M M N E R VA A gateway to Melbourne's research publications}

Minerva Access is the Institutional Repository of The University of Melbourne

Author/s:

Baker, S;Waycott, J;Carrasco, R;Hoang, T;Vetere, F

Title:

Exploring the Design of Social VR Experiences with Older Adults

Date:

2019

Citation:

Baker, S., Waycott, J., Carrasco, R., Hoang, T. \& Vetere, F. (2019). Exploring the Design of Social VR Experiences with Older Adults. Designing Interactive Systems, pp.303-315. ACM. https://doi.org/10.1145/3322276.3322361.

Persistent Link:

http://hdl.handle.net/11343/241847 